\title{
Strong cardiovascular prognostic implication of quantitative left atrial contractile function assessed by cardiac magnetic resonance imaging in patients with chronic hypertension
}

Matthew Kaminski ${ }^{1}$, Kevin Steel ${ }^{1}$, Michael Jerosch-Herold ${ }^{2}$, Maung Khin ${ }^{1}$, Sui Tsang ${ }^{1}$, Thomas Hauser ${ }^{3}$ and Raymond Y Kwong ${ }^{1 *}$

\begin{abstract}
Background: Progressive left ventricular (LV) diastolic dysfunction due to hypertension (HTN) alters left atrial (LA) contractile function in a predictable manner. While increased LA size is a marker of LV diastolic dysfunction and has been shown to be predictive of adverse cardiovascular outcomes, the prognostic significance of altered LA contractile function is unknown.
\end{abstract}

Methods: A consecutive group of patients with chronic hypertension but without significant valvular disease or prior MI underwent clinically-indicated CMR for assessment of left ventricular (LV) function, myocardial ischemia, or viability. Calculation of LA volumes used in determining LA emptying functions was performed using the biplane area-length method.

Results: Two-hundred and ten patients were included in this study. During a median follow-up of 19 months, 48 patients experienced major adverse cardiac events (MACE), including 24 deaths. Decreased LA contractile function $\left(\mathrm{LAEF}_{\text {Contractile }}\right)$ demonstrated strong unadjusted associations with patient mortality, non-fatal events, and all MACE. For every $10 \%$ reduction of $L A E F$ Contractile, unadjusted hazards to MACE, all-cause mortality, and non-fatal events increased by $1.8,1.5$, and 1.4-folds, respectively. In addition, preservation of the proportional contribution from LA contraction to total diastolic filling (Contractile/Total ratio) was strongly associated with lower MACE and patient mortality. By multivariable analyses, LAEF contractile was the strongest predictor in each of the best overall models of MACE, all-cause mortality, and non-fatal events. Even after adjustment for age, gender, left atrial volume, and LVEF, LAEF Contractile maintained strong independent associations with MACE $(p<0.0004)$, all-cause mortality $(p<0.0004)$, and non-fatal events $(p<0.0004)$.

Conclusions: In hypertensive patients at risk for left ventricular diastolic dysfunction, a decreased contribution of LA contractile function to ventricular filling during diastole is strongly predictive of adverse cardiac events and death.

\section{Background}

Left Ventricular (LV) diastolic dysfunction as a consequence of chronic hypertension is a prevalent condition associated with significant morbidity and mortality. Current strong prognostic markers that reflect diastolic dysfunction remain limited, but their identification may improve

\footnotetext{
* Correspondence: rykwong@partners.org

'Cardiovascular Division, Department of Medicine, Brigham and Women's

Hospital, Boston, Massachusetts, USA

Full list of author information is available at the end of the article
}

treatment planning and monitoring of patients with chronic hypertension. LA size reflects the duration and severity of exposure to increased diastolic filling pressures in the LV and is relatively load-independent. As LV diastolic impairment progresses, effective diastolic filling becomes increasingly dependent on LA contractile function until the LA contractile reserve can no longer meet the demand of elevated diastolic ventricular pressure[1-3]. While evidence exists that LA enlargement is a strong predictor of adverse cardiovascular outcomes in selected populations, there is

\section{Ciomed Central}


little data regarding the prognostic implication of altered left atrial contractile function in patients with chronic hypertension at risk of diastolic dysfunction. While 'ejection fraction' is the phrase used for reduction of ventricular cavity volume, 'emptying function' is more appropriate for reductions of atrial volume as the atria lack inflow valves and empty passively as well as actively. Cardiac magnetic resonance imaging (CMR) can quantify left atrial volume and emptying functions with sufficient temporal and spatial resolution and excellent reproducibility. Accordingly, this study aims to test the hypothesis that altered LA contractile emptying functions as measured by cine CMR can provide strong prognostic information in patients with chronic hypertension, beyond left atrial volume and other known risk predictors in this population.

\section{Methods \\ Patient Population}

We studied a consecutive series of patients with history of chronic hypertension medically treated for at least 6 months who were referred to undergo cardiac magnetic resonance imaging (CMR) for clinical purposes. Patients were referred either for a) evaluation for myocardial ischemia with stress CMR or b) assessment of regional and global left ventricular function and myocardial mass. Patients with any of the following were excluded: a) any evidence of myocardial infarction by history, medical record, or abnormal cardiac enzymes, b) any significant aortic or mitral valvular dysfunction (moderate or severe dysfunction by qualitative echocardiographic grading), and c) confirmed (by biopsy) myocarditis, infiltrative cardiomyopathy (including cardiac hemochromatosis, amyloidosis, or sarcoidosis), or pericardial disease. Other exclusion criteria included concurrent unstable angina, NYHA class IV heart failure, hemodynamic instability, claustrophobia precluding CMR, and metallic hazards. Patients with patterns of late gadolinium enhancement consistent with infiltrative cardiomyopathy or myocarditis were also excluded. The institutional ethics committee of the Brigham and Women's Hospital (Partners Healthcare system) approved the clinical follow-up activities of the study.

\section{Clinical Evaluation}

All patients underwent a detailed history immediately before the CMR. Hypertension, hypercholesterolemia, diabetes, and family history of premature CAD were defined by published criteria[4-7]. Significant smoking was defined by $>10$ pack-years of tobacco use. History of CAD included documented $>70 \%$ stenosis on angiography or history of coronary revascularization prior to CMR study.

\section{CMR Imaging}

All patients were studied supine in a $1.5 \mathrm{~T}$ CMR system (Signa ${ }^{\circledR} \mathrm{CV} / i$, GE Healthcare, USA) with a 4-element or 8-element phased-array surface coil. CMR study consisted of cine SSFP imaging (TR/TE 3.4/1.2ms, in-plane spatial resolution $1.6 \times 2 \mathrm{~mm}$, matrix $192 \times 160)$ of $\mathrm{LV}$ function and late gadolinium enhancement imaging (TR/TE 4.8/1.3ms, TI 200-300ms) for myocardial scar. All images were acquired using retrospective ECG gating and breath-holding. Cine and late enhancement imaging were obtained in 8-14 matching short-axis $(8 \mathrm{~mm}$ thick with $0 \mathrm{~mm}$ spacing) and 3 radial long-axis planes. The 3 radial long-axis planes were prescribed at 60 degrees apart. Typical view per segment during a cine SSFP acquisition was 12 yielding a temporal resolution of approximately $45 \mathrm{~ms}$ and maintaining a breath-hold of approximately 10-12 seconds for each slice location. A previously described segmented inversion-recovery pulse sequence for late enhancement imaging was used [8] starting at 15 minutes after cumulative $0.15 \mathrm{mmol} /$ $\mathrm{Kg}$ dose of gadolinium-DPTA. A single reader categorized late gadolinium enhancement as either typical infarction (involving the subendocardium) or atypical (subepicardial, patchy midwall or diffuse circumferential subendocardial pattern).

\section{CMR Quantitative Analysis of Left Atrial Volume and Emptying Function}

Quantitative Analysis of the left atrium is illustrated in Figure 1. To measure the left atrial dimensions, manual tracings were made of the left atrial area and long axis in the radial 2-chamber and 4-chamber views. For each radial view, tracings were performed at three phases: maximal LA volume just before mitral valve opening, minimal LA volume at mitral valve closure, and immediately prior to

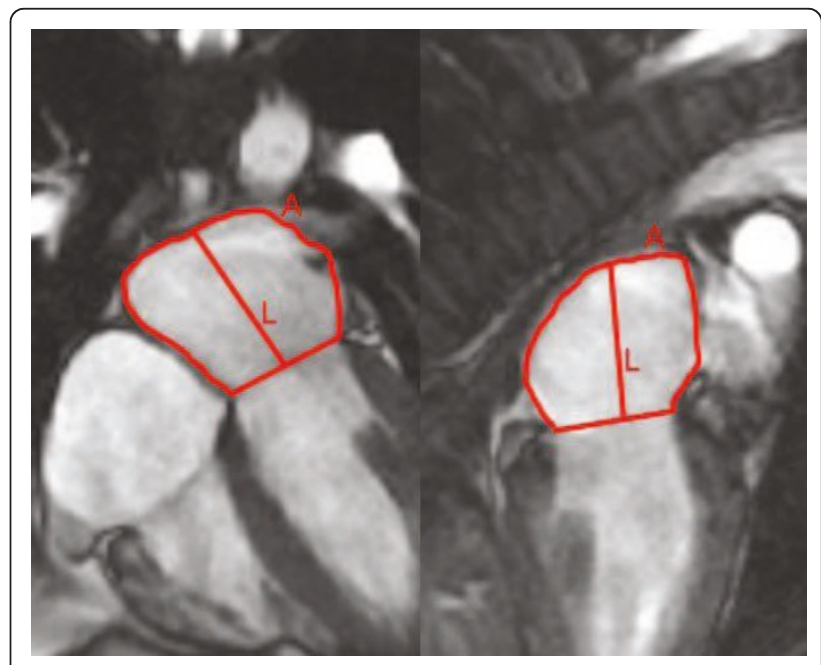

Figure 1 Measurement of left atrial area (A) and length (L) in 4-chamber and 2-chamber views used in the calculation of the left atrial volume indices (LAV) across different phases of the cardiac cycle. 
atrial contraction. At each phase, LA volume is calculated by the previously validated biplane area-length method[9] as follows: LA volume $(\mathrm{ml})=0.85^{*} \mathrm{~A}_{2 \mathrm{C}} \mathrm{C}_{4 \mathrm{C}} / \mathrm{L}$, where $\mathrm{A}_{2 \mathrm{C}}$ and $\mathrm{A}_{4 \mathrm{C}}$ are the LA areas on the 2-chamber and 4-chamber views, respectively, and $\mathrm{L}$ is the shorter long-axis length of the LA from either the 2-chamber or the 4-chamber views (Figure 1). Consistent with the recommendation of published reports[10], all LA volumes were normalized to the patient's body surface area in subsequent analyses. Total LA emptying volume was calculated as the difference between maximum $\left(\mathrm{LAV}_{\max }\right)$ and minimum LA volumes $\left(\mathrm{LAV}_{\mathrm{min}}\right)$. Total LA emptying volume was divided into LA passive emptying volume $\left(\mathrm{V}_{\text {Passive }}\right)$ and LA contractile volume $\left(\mathrm{V}_{\text {Contractile }}\right)$, in which $\mathrm{V}_{\text {Passive }}$ was calculated as the difference between $L A V_{\max }$ and the LA volume preceding atrial contraction $\left(\mathrm{LAV}_{\mathrm{ac}}\right)$ and $\mathrm{V}_{\mathrm{Con}-}$ tractile was calculated as the difference between $\mathrm{LAV}_{\mathrm{ac}}$ and $\mathrm{LAV}_{\text {min }}$. LA total, passive, and active emptying functions $\left(\mathrm{LAEF}_{\text {Total }}, \mathrm{LAEF}_{\text {Passive }}, \mathrm{LAEF}_{\text {Contractile }}\right)$ were calculated according to the following formulas:

$$
\begin{aligned}
& \mathrm{LAEF}_{\text {Total }}=\left(\mathrm{LAV}_{\text {max }}-\mathrm{LAV}_{\mathrm{ac}}-\mathrm{LAV}_{\text {min }}\right) * 100 / \mathrm{LAV}_{\text {max }} \\
& \mathrm{LAEF}_{\text {Passive }}=\left(\mathrm{LAV}_{\text {max }}-\mathrm{LAV}_{\mathrm{ac}}\right) * 100 / \mathrm{LAV}_{\text {max }} \\
& \mathrm{LAEF}_{\text {Contractile }}=\left(\mathrm{LAV}_{\mathrm{ac}}-\mathrm{LAV}_{\text {min }}\right) * 100 / \mathrm{LAV}_{\mathrm{ac}}
\end{aligned}
$$

In additon, we calculate the proportional contribution of LA contraction during diastole by calculating the following parameters:

$$
\begin{aligned}
\text { Contractile } / \text { Passive ratio } & =\mathrm{V}_{\text {Contractile }} / \mathrm{V}_{\text {Passive }} \\
\text { Contractile } / \text { Total ratio } & =\mathrm{V}_{\text {Contractile }} /\left(\mathrm{V}_{\text {Contractile }}+\mathrm{V}_{\text {Passive }}\right)
\end{aligned}
$$

While left atrial dimension (in millimeters) was made available to the attending physicians on the day of the CMR, all other quantitative measurements of the left atrium were not reported as part of the routine clinical care.

\section{CMR Quantitative Analysis of LV Function and Definition of Myocardial Ischemia}

All images were analyzed with specialized software (CineTool 2.80, General Electric Healthcare). We graded segmental systolic wall motion as normal or abnormal in each study and also graded segmental wall motion using a 4-point scale ( 1 = normokinesia, 2 = hypokinesia, $3=$ akinesia, and $4=$ dyskinesia) according to the standard 17-segment ACC/AHA nomenclature[11]. We interpreted late gadolinium myocardial enhancement (LGE) as present or absent. Details of segmental wall motion and LGE grading were as previously reported[12]. We manually traced epicardial and endocardial borders of matching short-axis cine locations at end systole and end-diastole to determine the LV ejection fraction
(LVEF), end-diastolic volume index (LVEDVI), end-systolic volume index (LVESVI), and the LV myocardial mass (end-diastole only)[13,14]. LVEF was measured by standard Simpson's Rule[14]. Presence of ischemia during dobutamine stress was defined by standard criteria of worsening regional wall motion by at least 1 grade, matching on short and long-axis cine, as published in prior reports[15]. Presence of ischemia during adenosine stress perfusion was according to prior publication, defined by existence of perfusion defect without infarction by LGE imaging[16].

\section{ECG Interpretation}

Resting 12-lead ECGs were obtained at a median of 1 day (interquartile range: 0-7 days) from CMR. We confirmed that no cardiac event or revascularization occurred between the collection of ECG and the CMR study. ECG interpretation was first performed by computer analysis followed by visual over-reading by a single reader blinded to the CMR results and the clinical outcome. ECG left atrial enlargement was defined by a terminal negative Pwave duration of $>40 \mathrm{~ms}$ and depth $\geq 1 \mathrm{~mm}$ measured on lead V1. We used the Sokolow-Lyon index to indicate LV hypertrophy on ECG[17].

\section{Follow-Up}

At least 6 months following the CMR, clinical information was obtained from patient telephone interviews, contacting patients' physicians, and hospital records. A standard questionnaire was used during telephone interview. Survival was obtained from the National Social Security Death Index in patients lost on first contact[18]. Major adverse cardiac events (MACE) included any of the following: 1) all-cause mortality, 2) new acute myocardial infarction, 3) unstable angina requiring hospitalization, and 4) development or progression of heart failure requiring hospitalization. New acute myocardial infarction was defined by significant elevation of serum troponins consistent with myocardial injury. Unstable angina was defined by new chest pain hospitalization without non-cardiac origin of chest pain, and with either angiographic coronary stenosis of $\geq 70 \%$ or ischemia on noninvasive imaging. Heart failure was defined by a need for hospitalization for new or worsening symptoms of heart failure as determined by the patient's cardiologist or primary internist. When a patient experienced $>1$ MACE, the first event was chosen. When $\geq 2 \mathrm{MACE}$ occurred simultaneously, the worse event was chosen (death $>\mathrm{MI}>$ unstable angina $>$ congestive heart failure).

\section{Statistical Analysis}

Baseline demographic differences, classified by the med-

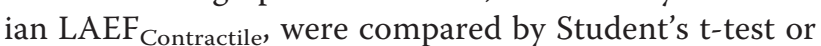
Fisher's exact test. Kaplan-Meier distributions for MACE, 
all-cause mortality, and nonfatal events were stratified by the median value of $\mathrm{LAEF}_{\text {Contractile }}$ and were compared by log-rank tests. We fitted Cox proportional-hazards models to estimate the likelihood ratio chi-square $\left(\mathrm{LR} \chi^{2}\right)$ and the unadjusted hazard ratios (HR) of all the variables. We also assessed the univariable association of the proportional contribution of LA contraction during diastole (Contractile/Passive and Passive/Total ratios) with MACE and other other events. To determine the set of variables that were most strongly associated with LAEFContractile, we performed linear regression using $\mathrm{LAEF}_{\text {Con- }}$ tractile as a continuous dependent variable, with all variables in Table 1 treated as independent variables. The prognostic association of LA size measurements and mechanical function estimates were also determined using similar analyses for all-cause mortality and nonfatal events specifically. We tested the interobserver agreement of $\mathrm{LAEF}_{\text {Contractile }}$ by Spearman correlation.

We performed 2 separate multivariable approaches to analyze the predictive value of CMR variables for MACE and all-cause mortality. In the first approach, we sought to determine the strongest set of variables that were associated with MACE, all-cause mortality, and non-fatal events, respectively, in this study cohort. We used a stepwise forward selection strategy and considered all clinical, ECG, or left ventricular variables as listed in Table 2. A p-value of 0.1 was used as criteria for variable inclusion or exclusion. In the second approach, we aimed to determine the prognostic association, if any, of $\mathrm{LAEF}_{\text {Contractile }}$ after adjustment to well-known risk predictors. Therefore, we sought to determine if $\mathrm{LAEF}_{\text {Contractile }}$ remained a significant predictor after adjustment to patient age, gender, minimal left atrial volume, and left ventricular ejection fraction. The validity of the proportional-hazards assumptions of this final model for MACE was again tested and was valid for all the variables in this model. All analyses were performed with SAS 9.1 (SAS Institute, Cary, N.C.) for Windows.

\section{Results}

\section{Baseline Characteristics}

From a consecutive series of 225 patients with a history of chronic hypertension, 3 (1\%) had unsuccessful CMR due to large body habitus or technical problem. Seven patients $(3 \%)$ had moderate or severe aortic or mitral valvular dysfunction and were excluded from the study. Five patients were lost to follow-up but were reported alive. The remaining 210 (123 male, mean age $52 \pm 16$ years) formed the study cohort and were followed for a median 19 months (range 6-50 months). Patients were referred to undergo stress CMR for evaluation of ischemia $(n=106)$ or for assessment of left ventricular function $(\mathrm{n}=104)$. Table 1 illustrates the demographical and ECG features of the entire study cohort and also data stratified by the median value of $\mathrm{LAEF}_{\text {Contractile }}(33 \%)$. Baseline average left ventricular ejection fraction at rest was low normal at $58 \pm 13 \%$. During the study period, 53 patients were referred to undergo coronary angiography at a median 34 days (interquartile range 1-12 days) from CMR study. Among them 38 patients (72\%) were found to have significant $(>70 \%)$ luminal stenosis. LAEF $_{\text {Contrac- }}$ tile dichotomized by its median level did not show correlation to the presence of angiographic coronary stenosis, history of percutaneous coronary intervention, or history of cardiac bypass surgery. Patients with LAEF Contractile below median value demonstrated a larger LA anteroposterior dimension $(42 \pm 9.1 \mathrm{~mm}$ vs. $38 \pm 6.2 \mathrm{~mm}, \mathrm{P}<$ 0.01 ), lower left ventricular ejection fraction ( $55 \pm 15 \%$ vs. $61 \pm 10 \%, \mathrm{P}<0.01)$, and higher left ventricular endsystolic volume index $\left(79 \pm 57 \mathrm{ml} / \mathrm{m}^{2}\right.$ vs. $65 \pm 41 \mathrm{ml} / \mathrm{m}^{2}$, $\mathrm{P}<0.05)$. There was high inter-observer correlation in quantifying LA volume (kappa statistic 0.83 ) across the three time phases of the cardiac cycle.

\section{Cardiovascular Outcome}

At a median follow-up of 19 months (range 6 to 47 months), 48 MACE occurred. Among them were 21 cases of death, 3 acute myocardial infarction, 12 cases of hospitalization for unstable angina, and 12 cases of congestive heart failure requiring hospitalization.

Relationship of Left Atrial Enlargement by ECG, LV Mass, and Left Atrial Volume and Mechanical Functions

Twenty patients (10\%) demonstrated left atrial enlargement on ECG. The presence of left atrial enlargement by ECG was only weakly associated with a higher than median $\operatorname{LAV}_{\min }(\mathrm{P}=0.045)$ and did not demonstrate significant correlation with above median $\mathrm{LAV}_{\max }$. Left atrial enlargement by ECG demonstrated poor sensitivity (16\%) but excellent specificity (93\%) in identifying patients with above median $\mathrm{LAV}_{\text {min }}$. Left atrial enlargement by ECG could not differentiate above or below median level of $\mathrm{LAEF}_{\text {Contractile. While LV mass }}(\mathrm{g})$ is slightly higher in patients with above median $\mathrm{LAV}_{\text {min }}(134 \pm 44$ vs. $152 \pm$ $55, \mathrm{P}=0.01)$, it was not significantly different among patients with above or below median level of $\mathrm{LAEF}_{\text {Contrac- }}$ tile. Left ventricular ejection fraction was negatively correlated with $\mathrm{LAV}_{\text {min }}(\mathrm{r}=-0.19, \mathrm{P}=0.008)$ and positively correlated with $\mathrm{LAEF}_{\text {Contractile }}(\mathrm{r}=0.15, \mathrm{p}=0.04)$. By multivariable linear regression, a history of atrial fibrillation on ECG, $L A V_{\text {min }}$, and left ventricular ejection fraction were most strongly predictive of $\mathrm{LAEF}_{\text {Contractile }}$

\section{Prognostic Association of Left Atrial Volume and Left Atrial Mechanical Function of the Cohort}

Univariable association of all variables with MACE, allcause mortality, and non-fatal events is listed in Table 2. In the current study cohort, presence of LVH on ECG 
Table 1 Demographic Characteristics of the Study Cohort

\begin{tabular}{|c|c|c|c|c|}
\hline & $\begin{array}{l}\text { All Patients } \\
(\mathrm{n}=210)\end{array}$ & $\begin{array}{l}\text { LA contractile function } \geq \text { median } \\
\qquad(\mathrm{n}=96)\end{array}$ & $\begin{array}{l}\text { LA contractile function }<\text { median } \\
\qquad(\mathrm{n}=114)\end{array}$ & P-value \\
\hline \multicolumn{5}{|l|}{ Clinical Characteristics } \\
\hline Age in years & $52 \pm 16$ & $52 \pm 16$ & $51 \pm 15$ & NS \\
\hline Female Gender (\%) & $87(41)$ & $42(44)$ & $45(39)$ & NS \\
\hline Resting Heart Rate & $71 \pm 15$ & $71 \pm 15$ & $71 \pm 15$ & NS \\
\hline Hx. of Diabetes (\%) & $71(34)$ & $32(33)$ & $39(34)$ & NS \\
\hline Hx. of Hypercholesterolemia (\%) & $138(65)$ & $60(63)$ & $78(68)$ & NS \\
\hline Heavy Tobacco Use (\%) & $56(27)$ & $24(25)$ & $32(28)$ & NS \\
\hline Hx of PAD (\%) & $21(10)$ & $6(6)$ & $15(13)$ & NS \\
\hline $\begin{array}{l}\text { Hx. of Percutaneous Coronary Intervention } \\
\text { (\%) }\end{array}$ & $25(12)$ & $12(13)$ & $13(11)$ & NS \\
\hline Hx. of Cardiac Bypass Surgery (\%) & $21(10)$ & $6(6)$ & $15(13)$ & NS \\
\hline $\begin{array}{l}\text { Hx. of Angiographic Coronary Stenosis } \\
\text { Before CMR (\%) }\end{array}$ & $45(21)$ & $22(23)$ & $23(20)$ & NS \\
\hline Any Hx. of CAD before CMR (\%) & $41(20)$ & $17(18)$ & $24(21)$ & NS \\
\hline Resting SBP & $145 \pm 25$ & $148 \pm 25$ & $142 \pm 24$ & NS \\
\hline Resting DBP & $75 \pm 13$ & $76 \pm 12$ & $75 \pm 15$ & NS \\
\hline \multicolumn{5}{|l|}{ Medication } \\
\hline Beta-blocker (\%) & $136(65)$ & $55(57)$ & $81(71)$ & 0.04 \\
\hline Calcium Blocker (\%) & $52(25)$ & $26(27)$ & $26(23)$ & NS \\
\hline $\begin{array}{l}\text { Angiotensin-converting enzyme inhibitor } \\
(\%)\end{array}$ & $103(49)$ & $51(53)$ & $52(46)$ & NS \\
\hline Aspirin (\%) & $116(55)$ & $51(53)$ & $65(57)$ & NS \\
\hline Nitrates (\%) & $23(11)$ & $9(9)$ & $14(12)$ & NS \\
\hline Digoxin (\%) & $4(2)$ & $1(1)$ & $3(3)$ & NS \\
\hline \multicolumn{5}{|l|}{$\underline{\text { Rest Electrocardiogram }}^{ \pm}$} \\
\hline Left Atrial Enlargement on ECG (\%) & $24(11)$ & $13(14)$ & $10(9)$ & NS \\
\hline Left Ventricular Hypertrophy on ECG & $17(8)$ & $7(7)$ & $10(9)$ & NS \\
\hline QRS duration (ms) & $100 \pm 25$ & $97 \pm 20$ & $102 \pm 27$ & NS \\
\hline Left bundle branch block (\%) & $19(9)$ & $9(9)$ & $10(9)$ & NS \\
\hline Right bundle branch block (\%) & $16(8)$ & $3(3)$ & $13(11)$ & 0.05 \\
\hline QTc interval & $439 \pm 39$ & $433 \pm 36$ & $443 \pm 40$ & 0.09 \\
\hline $\begin{array}{l}\text { Significant Q-waves by Minnesota Code } \\
\text { Criteria }\end{array}$ & $19(9)$ & $12(12)$ & $7(6)$ & NS \\
\hline \multicolumn{5}{|l|}{$\underline{C M R}$} \\
\hline Ao root dimen (mm) & $28 \pm 5$ & $28 \pm 4$ & $28 \pm 5$ & NS \\
\hline LVEF (\%) & $58 \pm 13$ & $61 \pm 10$ & $55 \pm 15$ & $<0.01$ \\
\hline LV mass (grams) & $142 \pm 50$ & $139 \pm 50$ & $145 \pm 51$ & NS \\
\hline LVEDD (mm) & $54 \pm 8$ & $53 \pm 8$ & $55 \pm 8$ & NS \\
\hline LVEDV index $\left(\mathrm{ml} / \mathrm{m}^{2}\right)$ & $164 \pm 59$ & $160 \pm 54$ & $167 \pm 63$ & NS \\
\hline LVESV index $\left(\mathrm{ml} / \mathrm{m}^{2}\right)$ & $73 \pm 50$ & $65 \pm 41$ & $79 \pm 57$ & 0.04 \\
\hline LA Volume index - end-systole $\left(\mathrm{ml} / \mathrm{m}^{2}\right)$ & $51 \pm 20$ & $45 \pm 16$ & $57 \pm 21$ & $<0.0001$ \\
\hline $\begin{array}{l}\text { LA Volume index - before atrial contraction } \\
\left(\mathrm{ml} / \mathrm{m}^{2}\right)\end{array}$ & $41 \pm 18$ & $72 \pm 31$ & $88 \pm 38$ & 0.001 \\
\hline LA Volume index - end-diastole $\left(\mathrm{ml} / \mathrm{m}^{2}\right)$ & $30 \pm 19$ & $21 \pm 9$ & $39 \pm 22$ & $<0.0001$ \\
\hline LAEF $_{\text {Passive }}(\%)$ & $19 \pm 12$ & $20 \pm 12$ & $18 \pm 12$ & NS \\
\hline LAEF $_{\text {Contractile }}(\%)$ & $32 \pm 15$ & $42 \pm 7$ & $21 \pm 13$ & $<0.0001$ \\
\hline LAEF $_{\text {Total }}(\%)$ & $44 \pm 16$ & $54 \pm 9$ & $34 \pm 15$ & $<0.0001$ \\
\hline Contractile/Passive Ratio & $3.8 \pm 19$ & $5.1 \pm 25$ & $2.4 \pm 8$ & $<0.0001$ \\
\hline Contractile/Total Ratio & $0.57 \pm 0.29$ & $0.64 \pm 0.18$ & $0.50 \pm 0.37$ & $<0.0001$ \\
\hline
\end{tabular}


Table 2 Univariable Association of Variables with All MACE, All-Cause Mortality, and Non-fatal Events

\begin{tabular}{|c|c|c|c|c|c|c|c|c|c|}
\hline & \multicolumn{3}{|c|}{ All MACE $(\mathrm{N}=48)$} & \multicolumn{3}{|c|}{ All-Cause Mortality $(\mathrm{N}=21)$} & \multicolumn{3}{|c|}{ Non-Fatal Events $(\mathrm{N}=27)$} \\
\hline & $\operatorname{LR} \chi^{2}$ & $\mathrm{HR}(95 \% \mathrm{Cl})$ & P-value & $\mathrm{LR} \chi^{2}$ & $\mathrm{HR}(95 \% \mathrm{Cl})$ & P-value & $\mathrm{LR} \chi^{2}$ & HR & P-value \\
\hline Age (years) & 0.41 & $1.01(0.99-1.03)$ & NS & 0.51 & $1.01(0.98-1.04)$ & NS & 0.02 & $1.00(0.97-1.03)$ & NS \\
\hline Female Gender & 2.12 & $1.63(0.84-3.15)$ & NS & 4.65 & $2.72(1.10-6.73)$ & 0.03 & 0.01 & $1.04(0.41-2.63)$ & NS \\
\hline Body Mass Index (m/ $\left.\mathrm{kg}^{2}\right)$ & 1.62 & $0.96(0.91-1.02)$ & NS & 0.19 & $0.98(0.91-1.06)$ & NS & 2.19 & $0.94(0.86-1.02)$ & NS \\
\hline Hx. Percutaneous Coronary Intervention & 3.21 & $2.07(0.93-4.56)$ & 0.07 & 0.54 & $1.51(0.51-4.48)$ & NS & 1.97 & $2.25(0.73-6.98)$ & NS \\
\hline Hx. Coronary Bypass Surgery & 3.69 & $2.25(0.96-5.13)$ & 0.05 & 0.23 & $1.35(0.40-4.58)$ & NS & 3.44 & $2.87(0.94-8.74)$ & 0.06 \\
\hline Diabetes & 0.49 & $1.27(0.65-2.47)$ & NS & 0.01 & $1.00(0.41-2.42)$ & NS & 0.07 & $1.14(0.44-2.96)$ & NS \\
\hline Hypercholesterolemia & 0.10 & $1.12(0.54-2.34)$ & NS & 0.82 & $0.67(0.28-1.61)$ & NS & 1.53 & $2.19(0.63-7.59)$ & NS \\
\hline Hx. Heavy Smoking & 0.60 & $1.31(0.66-2.57)$ & NS & 0.17 & $0.82(0.31-2.12)$ & NS & 1.09 & $1.65(0.64-4.22)$ & NS \\
\hline Family Hx. CAD & 1.00 & $0.66(0.29-1.49)$ & NS & 0.33 & $0.77(0.32-1.85)$ & NS & 0.47 & $0.67(0.21-2.11)$ & NS \\
\hline \multicolumn{10}{|l|}{ Medications } \\
\hline Beta-blocker Use & 4.68 & $2.63(1.10-6.33)$ & 0.03 & 4.27 & $4.65(1.08-19.96)$ & 0.04 & 1.08 & $1.80(0.59-5.49)$ & NS \\
\hline Calcium Channel Blocker Use & 1.08 & $1.44(0.72-2.89)$ & NS & 0.01 & $0.95(0.35-2.59)$ & NS & 1.66 & $1.87(0.72-4.83)$ & NS \\
\hline ACE Inhibitor Use & 0.05 & $0.93(0.48-1.79)$ & NS & 0.01 & $0.98(0.42-2.31)$ & NS & 0.74 & $0.66(0.25-1.70)$ & NS \\
\hline Aspirin Use & 0.97 & $1.42(0.71-2.83)$ & NS & 0.04 & $0.92(0.39-2.18)$ & NS & 1.43 & $1.88(0.67-5.27)$ & NS \\
\hline \multicolumn{10}{|l|}{$\underline{\text { ECG Variables }}$} \\
\hline History of Atrial Fibrillation on ECG & 2.17 & $2.22(0.77-6.43)$ & NS & 1.22 & $2.30(0.53-10.05)$ & NS & 0.66 & $1.87(0.41-8.46)$ & NS \\
\hline Left Atrial Enlargement on ECG & 0.01 & $1.04(0.36-2.96)$ & NS & 0.62 & $0.45(0.06-3.45)$ & NS & 0.61 & $1.65(0.47-5.82)$ & NS \\
\hline LVH Voltage and Strain & 1.92 & $1.97(0.75-5.14)$ & NS & $* *$ & $* *$ & NS & 7.58 & $4.43(1.54-12.77)$ & $<0.01$ \\
\hline Significant Q Waves & 0.64 & $1.53(0.54-4.39)$ & NS & 3.73 & $3.00(0.98-19.12)$ & 0.05 & $* *$ & $* *$ & $* *$ \\
\hline QRS Interval > $120 \mathrm{~ms}$ & 0.39 & $0.72(0.25-2.04)$ & NS & 0.29 & $0.67(0.15-2.91)$ & NS & 0.01 & $1.08(0.31-3.77)$ & NS \\
\hline Left Bundle Branch Block & 1.21 & $1.71(0.66-4.44)$ & NS & 0.05 & $1.18(0.27-5.15)$ & NS & 3.31 & $2.84(0.92-8.74)$ & 0.07 \\
\hline Right Bundle Branch Block & 0.23 & $0.70(0.17-2.94)$ & NS & 0.28 & $1.49(0.34-6.49)$ & NS & $* *$ & $* *$ & $* *$ \\
\hline Significant ST Changes & 1.61 & $1.72(0.74-3.98)$ & NS & 0.06 & $0.83(0.19-3.62)$ & NS & 3.05 & $2.55(0.89-7.32)$ & 0.08 \\
\hline Significant $T$ Changes & 0.03 & $1.08(0.47-2.49)$ & NS & 0.10 & $1.19(0.39-3.62)$ & NS & 0.07 & $0.84(0.24-2.94)$ & NS \\
\hline Prolonged corrected QT Interval & 5.15 & $2.23(1.12-4.47)$ & 0.02 & 1.42 & $1.76(0.70-4.43)$ & NS & 4.58 & $2.99(1.10-8.14)$ & 0.03 \\
\hline \multicolumn{10}{|l|}{ CMR Variables of the LV } \\
\hline LVEDD (mm) & 0.70 & $1.02(0.98-1.06)$ & NS & 2.93 & $0.95(0.90-1.01)$ & 0.09 & 8.32 & $1.08(1.02-1.14)$ & $<0.01$ \\
\hline LVEF (per 10\%) & 2.59 & $0.84(0.68-1.04)$ & NS & 0.04 & $1.03(0.75-1.42)$ & NS & 5.3 & $0.71(0.54-0.95)$ & 0.02 \\
\hline LVEDVI (per $10 \mathrm{ml} / \mathrm{m}^{2}$ ) & 0.52 & $1.02(0.97-1.07)$ & NS & 4.06 & $0.89(0.80-1.00)$ & NS & 6.97 & $1.08(1.02-1.13)$ & $<0.01$ \\
\hline LVESVI (per $10 \mathrm{ml} / \mathrm{m}^{2}$ ) & 3.66 & $1.05(1.00-1.10)$ & 0.06 & 1.00 & $0.94(0.82-1.07)$ & NS & 10.35 & $1.09(1.03-1.14)$ & 0.001 \\
\hline LV Mass (gram) & 0.01 & $1.00(0.99-1.01)$ & NS & 3.26 & $0.99(0.98-1.00)$ & 0.07 & 1.71 & $1.00(1.00-1.01)$ & NS \\
\hline Segmental Wall Motion Abnormality & 1.18 & $1.49(0.73-3.06)$ & NS & 0.66 & $1.49(0.57-3.93)$ & NS & 0.17 & $1.24(0.44-3.49)$ & NS \\
\hline Abnormal LV LGE & 6.33 & $2.47(1.22-5.00)$ & NS & 2.12 & $2.08(0.78-5.59)$ & NS & 3.91 & $2.69(1.01-7.18)$ & 0.05 \\
\hline \multicolumn{10}{|l|}{ CMR Variables of the LA } \\
\hline Anteroposterior LA Dimension (mm) & 4.64 & $1.05(1.00-1.09)$ & 0.03 & 1.01 & $1.03(0.97-1.10)$ & NS & 4.45 & $1.06(1.00-1.12)$ & 0.03 \\
\hline$L A V_{\max }\left(\mathrm{ml} / \mathrm{m}^{2}\right)$ & 2.50 & $1.01(1.00-1.03)$ & NS & 1.60 & $1.01(0.99-1.04)$ & NS & 2.94 & $1.02(1.00-1.04)$ & 0.09 \\
\hline $\mathrm{LAV}_{\mathrm{ac}}\left(\mathrm{ml} / \mathrm{m}^{2}\right)$ & 3.16 & $1.02(1.00-1.03)$ & 0.08 & 2.79 & $1.02(1.00-1.04)$ & 0.10 & 2.99 & $1.02(1.00-1.04)$ & 0.08 \\
\hline$L A V_{\min }\left(\mathrm{ml} / \mathrm{m}^{2}\right)$ & 6.28 & $1.02(1.00-1.03)$ & 0.01 & 6.48 & $1.02(1.00-1.03)$ & 0.01 & 3.84 & $1.02(1.00-1.03)$ & 0.05 \\
\hline LAEF $_{\text {Passive }}$ (per $10 \%$ reduction) & 0.47 & $1.12(0.81-1.57)$ & NS & 0.39 & $1.16(0.73-1.86)$ & NS & 0.46 & $1.17(0.74-1.86)$ & NS \\
\hline $\operatorname{LAEF}_{\text {Contractile }}$ (per 10\% reduction) & 18.84 & $1.75(1.36-2.24)$ & $<0.0001$ & 14.17 & $1.46(1.20-1.77)$ & $<0.001$ & 6.94 & $1.35(1.08-1.70)$ & $<0.01$ \\
\hline $\mathrm{LAEF}_{\text {Total }}$ (per $10 \%$ reduction) & 10.91 & $1.45(1.16-1.82)$ & $<0.001$ & 10.80 & $1.38(1.14-1.68)$ & 0.001 & 5.05 & $1.29(1.03-1.60)$ & 0.02 \\
\hline Contractile/Passive ratio & 10.64 & $0.94(0.91-0.98)$ & $<0.001$ & 1.47 & $0.88(0.72-1.08)$ & NS & 11.9 & $0.93(0.90-0.97)$ & $<0.001$ \\
\hline Contractile/Total Ratio & 10.48 & $0.39(0.24-0.64)$ & $<0.001$ & 8.75 & $0.21(0.08-0.59)$ & $<0.01$ & 1.21 & $0.48(0.13-1.77)$ & NS \\
\hline
\end{tabular}

* Too Few Events for Analysis.

did not demonstrate significant association with MACE or mortality but was associated with non-fatal cardiac events (HR 4.43, 95\% CI 1.54-12.77, $\mathrm{P}<0.01$ ). LV mass measurement did not demonstrate significant association with MACE. Decreasing left ventricular ejection fraction, increasing $L A V_{\text {min }}$ or $L A V_{\text {max }}$, and presence of late gadolinium myocardial enhancement were all associated with non-fatal events but did not achieved significant association with MACE or mortality. LAV $V_{\text {min }}$ (in

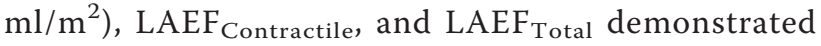


strong association with patient mortality, non-fatal events, and all MACE. Figure 2 illustrates the KaplanMeier curves demonstrating the time-to-event distributions for MACE, all-cause mortality, and non-fatal events, stratified by the median level of LAEF Contractile (Figure $2 \mathrm{~A}-\mathrm{C}$ ). The prognostic association of $\mathrm{LAEF}_{\mathrm{Con}-}$ tractile with all MACE maintained its significance regardless of the presence or absence of ECG finding of LVH (Figure 2D). For every $10 \%$ reduction of LAEF $_{\text {Contractile, }}$ unadjusted hazards to MACE, all-cause mortality, and non-fatal events increased by $1.8,1.5$, and 1.4-folds, respectively. By similar argument, preservation of the proportional contribution of diastolic filling by atrial contractile, as quantified by the Contractile/Passive or Contractile/Total ratio, demonstrated strong association with favorable outcome to MACE (unadjusted HR 0.94 and 0.39 , respectively, both $\mathrm{P}=0.001$ ), with a preserved Contractile/Total ratio portended to lower likelihood to mortality (HR to mortality $0.21, \mathrm{P}=0.003$ ). On the contrary, LA antero-posterior dimension only demonstrated a weak unadjusted prognostic association with non-fatal events but not with all-cause mortality (Table 2).

In the first multivariable approach, stepwise forward selection strategy demonstrated that $\mathrm{LAEF}_{\text {Contractile }}$ was the strongest multivariable predictor in each of the best overall models of MACE, all-cause mortality, and nonfatal events. For MACE prediction, $\mathrm{LAEF}_{\text {Contractile, his- }}$ tory of cardiac bypass surgery, and left bundle branch block on ECG were selected to form the best overall model. Figure 3 presents the model $\mathrm{LR} \chi^{2}$ of the selected multivariate variables in each of the best overall models for MACE, all-cause mortality, and non-fatal events, respectively (Figure $3 \mathrm{~A}-\mathrm{C}$ ). In the second multivariable approach, adjusted to the patients' age, gender, and LV ejection fraction, $\mathrm{LAEF}_{\text {Contractile }}$ maintained strong and independent prognostic association with MACE (model $\mathrm{LR} \chi^{2}$ increased from 4.99 to $22.02, \mathrm{P}<0.0001$ ), allcause mortality (model $\mathrm{LR} \chi^{2}$ increased from 4.54 to 15.88, $\mathrm{P}<0.0001$ ), and non-fatal events (model LR $\chi^{2}$ increased from 5.05 to $10.58, \mathrm{P}=0.005$ ).

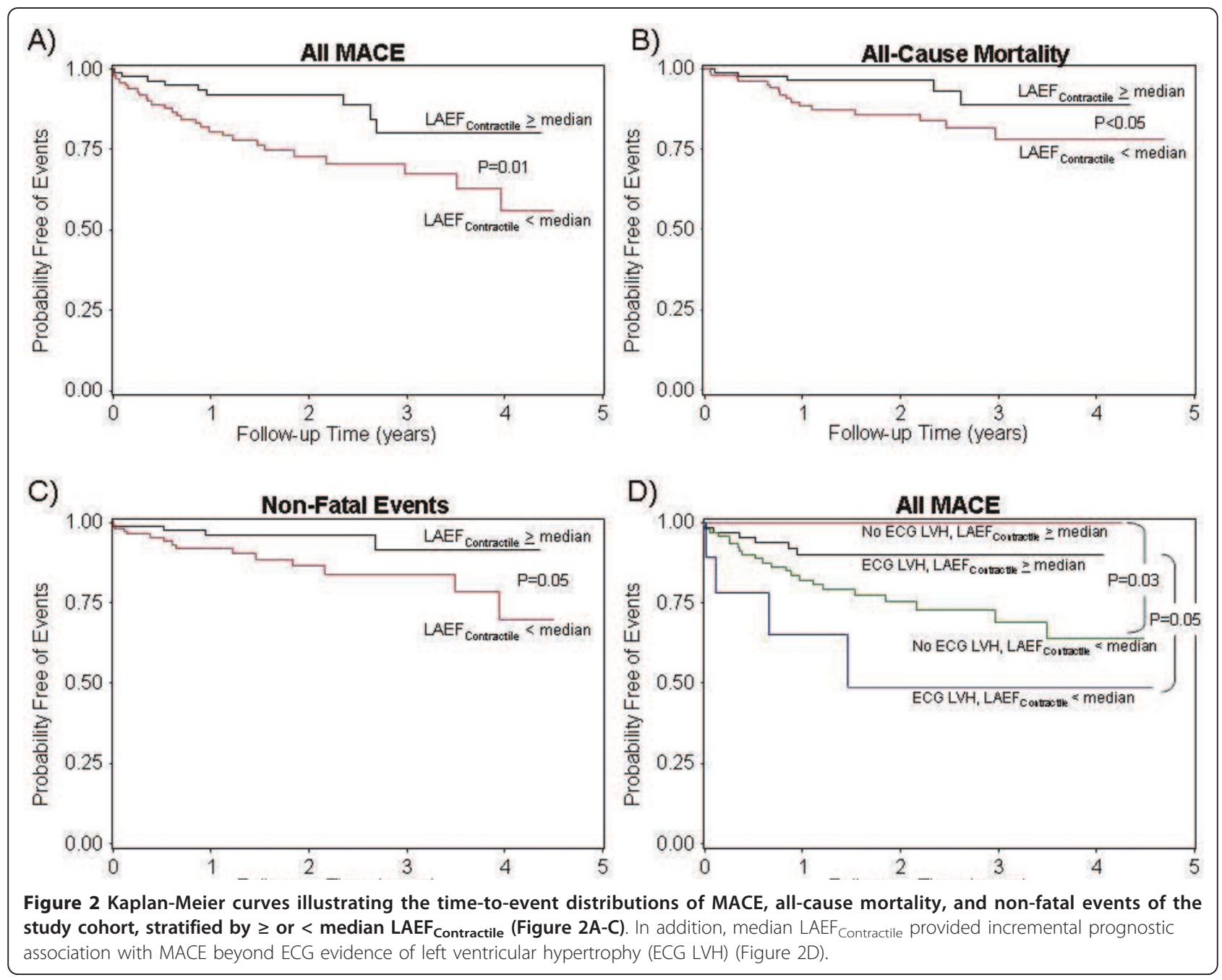




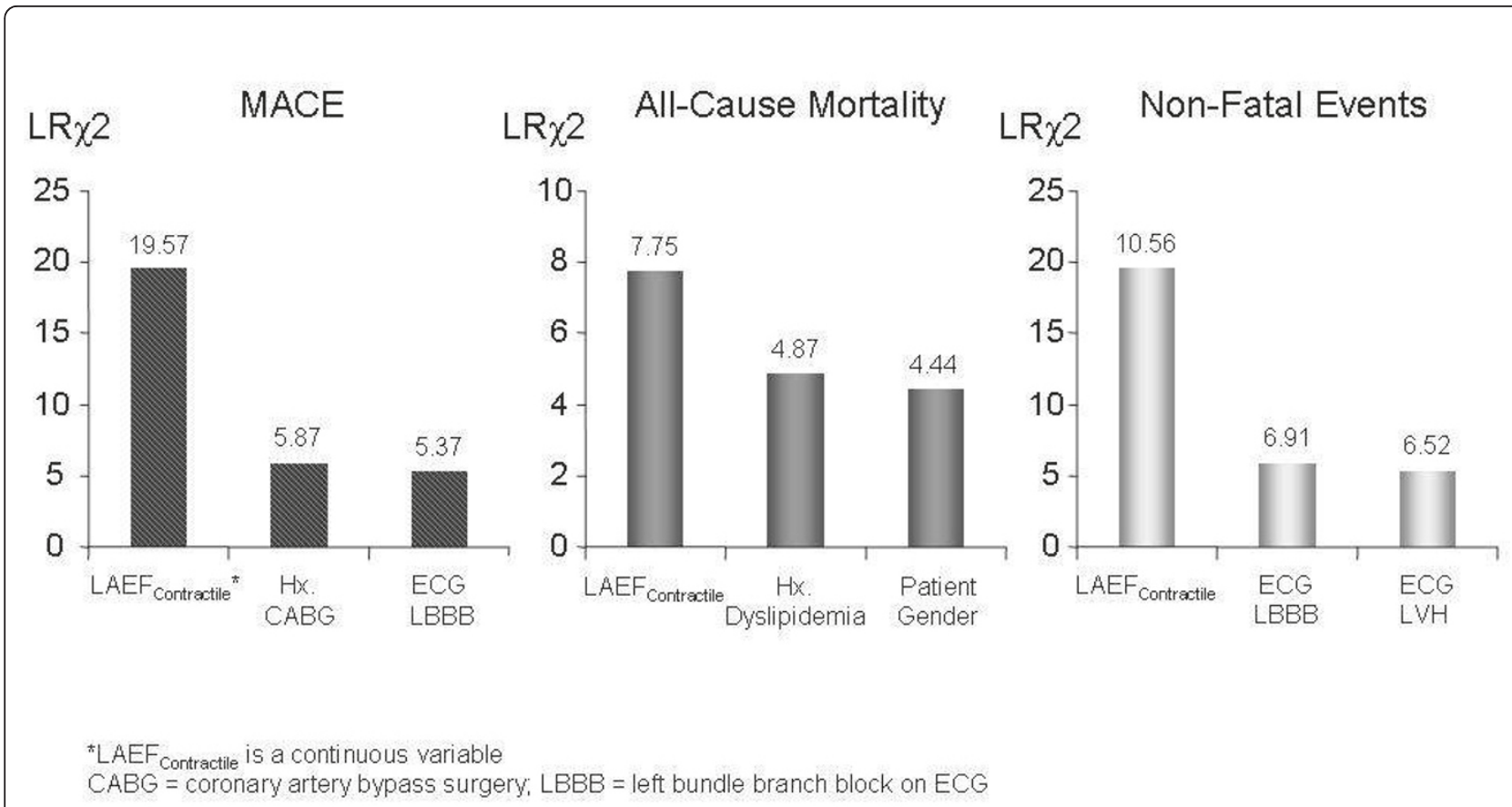

Figure 3 Multivariable Best Overall Models for MACE, All-Cause Mortality, and Non-Fatal Events. The respective LR $\chi^{2}$ of the predictors in each model is illustrated by the number on top of each column.

\section{Myocardial Ischemia and Left Atrial Mechanical Function} One hundred and six patients (50\%) of the cohort underwent CMR for evaluation of myocardial ischemia. Among them 52 (49\%) underwent dobutamine stress cine and 54 (51\%) underwent adenosine stress perfusion imaging. Nineteen (17\%) of the 106 patients were found to have myocardial ischemia during stress CMR. Twenty-six (25\%) of the 106 patients experienced MACE during study follow-up, among them 11 died. Mean LAEF $_{\text {Contractile was not significantly different }}$ between patients with or without myocardial ischemia $(33 \pm 11$ vs. $35 \pm 11, P=0.38)$. In the subgroup of patients who underwent CMR ischemia evaluation, presence of ischemia did not demonstrate any significant association with MACE, all-cause mortality, and nonfatal events (model $\mathrm{LR} \chi^{2} 0.90,0.08$, and 0,07, respectively). When presence of myocardial ischemia was entered into a model that contains $\mathrm{LAEF}_{\text {Contractile, the }}$ significant associations of $\mathrm{LAEF}_{\text {Contractile with MACE }}$ and all-cause mortality, respectively, were not altered (hazard ratios of $\mathrm{LAEF}_{\text {Contractile }}$ adjusted to ischemia, 1.97 and 2.17; $\mathrm{P}=0.0009$ and 0.004 , respectively).

\section{Discussion}

In this present study of hypertensive patients without significant valvular disease or myocardial infarction, a decreased contribution of LA contractile function to ventricular filling during diastole was strongly predictive of
MACE, mortality, and non-fatal events. We found that in these patients, proportional contribution of the LA contraction as measured by the LA Contractile/Total ratio are strongly associated with MACE and patient mortality. These observations were true even after adjustment for age, gender, LA volume and left ventricular ejection fraction. This postulates that altered LA mechanical contractile function by CMR in patients with or at risk for diastolic dysfunction from hypertension provides unique and independent prognostic value to adverse cardiovascular outcomes. To our knowledge, this is the first study which demonstrates a relationship between altered LA contractile function and CV outcomes, including mortality, in hypertensive patients.

In normal humans, passive emptying of the LA (a combination of the reservoir and conduit functions of the LA) during ventricular diastole contributes $75-80 \%$ of LV filling. LA contractile function normally contributes approximately $<25 \%$ of the total LV filling volume[19], but becomes increasing important to LV diastolic filling as ventricular stiffness progresses. In the mild stage of impaired LV relaxation, the LA contractile function increases and can contribute up to $38 \%$ of LV filling[1]. However, as LV stiffness progresses to moderate or severe range with marked increases in LV filling pressures, the contribution of LA contraction falls below the normal contribution, and the LA becomes mainly a passive conduit for ventricular filling $[3,10]$. Given what is known 
about the physiology of LA mechanical function, the present findings would seem to suggest that the increased risk of MACE and mortality might be conferred mostly on those patients with more severe (i.e. grade III or IV) restrictive LV diastolic dysfunction and thus decreased LA contractile function. Indeed, although patients in the general population with all grades of diastolic function are at increased risk of adverse CV outcomes and mortality, the risk is most pronounced in those patients with restrictive (grade III or IV) LV diastolic function [20]. This is also consistent with the known limited ability of the LA to reflect changes in mild or moderate LV diastolic dysfunction. Pritchett et al. has previously reported that in the general population, indexed LA volume has the highest sensitivity and specificity for the detection of severe (grade III or IV) LV diastolic dysfunction [21]. The same may be true of LA contractile function.

In this study, CMR is able to quantify LA contractile function at high spatial resolution for border delineation and adequate temporal resolution to capture LA motion throughout the cardiac cycle. We demonstrated such LA quantitative results are highly reproducible. While echocardiographic studies have assessed the relationship between LA volume and clinical outcomes, the prognostic relationship between the different components of LA mechanical function and clinical outcomes has not been well studied. We postulate that exposure of the LA myocardium to elevated left ventricular diastolic pressure can result in LA myopathy which can be quantified by LAEFContractile utilizing CMR as in the current study. Conformed to the existing body of echocardiographic literature, we used planar images of the LA acquired along its long axis and then used two dimensional measurements and standardized formulas to calculate LA volumes by the biplane area length method. It is therefore conceivable that the CMR findings of our study can be applied to similar patients undergoing echocardiographic assessment.

LA size has long been reported to be a sensitive and load-independent marker of both the severity and duration of LV diastolic dysfunction[22-24]. There is substantial and growing evidence pointing to the clear prognostic value of LA size in selected clinical populations. In the general population, increased LA volume has been shown to predict atrial fibrillation [23-28] stroke [22,29], and incidence of heart failure [30,31]. In addition, several authors have demonstrated the role of increased LA volume in predicting mortality in populations with pre-existing cardiovascular diseases, such as post-myocardial infarction or with LV dysfunction [32-35]. We believe that this study adds to this knowledge by showing that in patients with chronic hypertension, LA contractile function represents an additional prognostic tool by characterizing the burden of underlying diastolic dysfunction not assessed by current imaging techniques. Moreover, this study demonstrates the feasibility of using CMR to assess the LA in the clinical setting to predict cardiovascular outcomes.

\section{Limitations}

There are a number of study limitations. This study was a retrospective referral-based cohort study at a single center. All of the subjects studied had been referred for CMR examination, while the clinical indications of such were appropriate, the generalizability to other populations with hypertension may be limited. Only $50 \%$ of the current study cohort underwent stress CMR evaluation for myocardial ischemia. While we found that presence of myocardial ischemia did not seem to alter the prognostic significance of LA contractile function with the clinical outcomes, the current report does not have the study design or power to properly address whether myocardial ischemia could have been a confounding variable. The method of LA volume quantitation in this study can only provide estimates of LA volumes since it has not been validated against the axial stacked volumetric imaging. The study is also limited by its sample size and the small number of patients who experienced MACE, therefore some degree of model overfitting in the multivariable analyses may have occurred. For this reason, confirmation of the current study findings and exploration of potential therapeutic implications that can alter LA mechanics are necessary in a larger prospective study. Finally, concurrent load-independent measures of LV diastolic function such as tissue Doppler velocity are not available in the current study, thus prohibiting probing in the mechanism of the significant prognostic findings being reported in the current study. Such association of LA contractile function with imaging markers of diastolic dysfunction and serum markers such as naturetic peptide levels[36], will need to be studied prospectively.

\section{Conclusions}

In patients with a history of chronic hypertension, a decreased contribution of LA contractile function to ventricular filling during diastole appears to be a novel predictor of adverse cardiac events and death.

\section{Acknowledgements}

The authors would like to thank Vi Nguyen for her expertise in performing the CMR studies. Raymond Kwong, MD receives salary support from the National Institutes of Health (RO1HL091157).

\section{Author details}

${ }^{1}$ Cardiovascular Division, Department of Medicine, Brigham and Women's Hospital, Boston, Massachusetts, USA. ²Department of Radiology, Brigham and Women's Hospital, Boston, Massachusetts, USA. ${ }^{3}$ Cardiovascular Division, Beth Israel Deaconess Medical Center, Boston, Massachusetts, USA.

\section{Authors' contributions}

MK performed and MJH helped in post-processing and off-line analyses of the CMR images. MK, and KS collected clinical follow-up data in all of the patients. ST and TH participated in the design of the study, assisted in data 
entry, and performed the statistical analysis. RK conceived of the study, participated in its design, oversaw data acquisition at all levels, and helped to draft and revise the manuscript. All authors read and approved the final manuscript.

\section{Competing interests}

The authors declare that they have no competing interests.

Received: 26 November 2010 Accepted: 15 August 2011

Published: 15 August 2011

\section{References}

1. Appleton CP, Hatle LK, Popp RL: Relation of transmitral flow velocity patterns to left ventricular diastolic function: new insights from a combined hemodynamic and Doppler echocardiographic study. J Am Coll Cardiol 1988, 12(2):426-40.

2. Thomas L, Levett K, Boyd A, Leung DY, Schiller NB, Ross DL: Compensatory changes in atrial volumes with normal aging: is atrial enlargement inevitable? J Am Coll Cardiol 2002, 40(9):1630-5.

3. Prioli A, Marino P, Lanzoni L, Zardini P: Increasing degrees of left ventricular filling impairment modulate left atrial function in humans. Am J Cardiol 1998, 82(6):756-61.

4. Whelton PK: Epidemiology of hypertension. Lancet 1994 344(8915):101-6.

5. Third Report of the National Cholesterol Education Program (NCEP) Expert Panel on Detection, Evaluation, and Treatment of High Blood Cholesterol in Adults (Adult Treatment Panel III) final report. Circulation 2002, 106(25):3143-421.

6. Classification and diagnosis of diabetes mellitus and other categories of glucose intolerance. National Diabetes Data Group. Diabetes 1979, 28(12):1039-57.

7. Summary of the second report of the National Cholesterol Education Program (NCEP) Expert Panel on Detection, Evaluation, and Treatment of High Blood Cholesterol in Adults (Adult Treatment Panel II). JAMA 1993, 269(23):3015-23.

8. Simonetti OP, Kim RJ, Fieno DS, Hillenbrand HB, Wu E, Bundy JM, Finn JP, Judd RM: An improved MR imaging technique for the visualization of myocardial infarction. Radiology 2001, 218(1):215-23.

9. Lang RM, Bierig M, Devereux RB, Flachskampf FA, Foster E, Pellikka PA, Picard MH, Roman MJ, Seward J, Shanewise JS, Solomon SD, Spencer KT, Sutton MS, Stewart WJ: Recommendations for chamber quantification: a report from the American Society of Echocardiography's Guidelines and Standards Committee and the Chamber Quantification Writing Group, developed in conjunction with the European Association of Echocardiography, a branch of the European Society of Cardiology. J Am Soc Echocardiogr 2005, 18(12):1440-63.

10. Abhayaratna WP, Seward JB, Appleton CP, Douglas PS, Oh JK, Tajik AJ, Tsang TS: Left atrial size: physiologic determinants and clinical applications. J Am Coll Cardiol 2006, 47(12):2357-63.

11. Cerqueira MD, Weissman NJ, Dilsizian V, Jacobs AK, Kaul S, Laskey WK, Pennell DJ, Rumberger JA, Ryan T, Verani MS: Standardized myocardial segmentation and nomenclature for tomographic imaging of the heart: a statement for healthcare professionals from the Cardiac Imaging Committee of the Council on Clinical Cardiology of the American Heart Association. Circulation 2002, 105(4):539-42.

12. Kwong RY, Chan AK, Brown KA, Chan CW, Reynolds HG, Tsang S, Davis RB: Impact of unrecognized myocardial scar detected by cardiac magnetic resonance imaging on event-free survival in patients presenting with signs or symptoms of coronary artery disease. Circulation 2006, 113(23):2733-43

13. Salton CJ, Chuang ML, O'Donnell CJ, Kupka MJ, Larson MG, Kissinger KV Edelman RR, Levy D, Manning WJ: Gender differences and normal left ventricular anatomy in an adult population free of hypertension. A cardiovascular magnetic resonance study of the Framingham Heart Study Offspring cohort. J Am Col ICardiol 2002, 39(6):1055-60.

14. Alfakih K, Reid S, Jones T, Sivananthan M: Assessment of ventricular function and mass by cardiac magnetic resonance imaging. Eur Radiol 2004, 14(10):1813-22

15. Nagel E, Lehmkuhl HB, Bocksch W, Klein C, Vogel U, Frantz E, Ellmer A, Dreysse $S$, Fleck E: Noninvasive diagnosis of ischemia-induced wall motion abnormalities with the use of high-dose dobutamine stress MRI: comparison with dobutamine stress echocardiography. Circulation 1999, 99(6):763-70.

16. Steel K, Broderick R, Gandla V, Larose E, Resnic F, Jerosch-Herold M, Brown KA, Kwong RY: Complementary Prognostic Values of Stress Myocardial Perfusion and Late Gadolinium Enhancement Imaging by Cardiac Magnetic Resonance in Patients With Known or Suspected Coronary Artery Disease. Circulation 2009 [http://circ.ahajournals.org/ content/120/14/1390.full]

17. Okin PM, Devereux RB, Jern S, Kjeldsen SE, Julius S, Nieminen MS, Snapinn S, Harris KE, Aurup P, Edelman JM, Wedel H, Lindholm LH, Dahlof B: Regression of electrocardiographic left ventricular hypertrophy during antihypertensive treatment and the prediction of major cardiovascular events. JAMA 2004, 292(19):2343-9.

18. Davis KB, Fisher L, Gillespie MJ, Pettinger M: A test of the National Death Index using the Coronary Artery Surgery Study (CASS). Control Clin Trials 1985, 6(3):179-91.

19. Mitchell $J H$, Shapiro $W$ : Atrial function and the hemodynamic consequences of atrial fibrillation in man. Am J Cardiol 1969, 23(4):556-67.

20. Redfield MM, Jacobsen SJ, Burnett JC Jr, Mahoney DW, Bailey KR, Rodeheffer RJ: Burden of systolic and diastolic ventricular dysfunction in the community: appreciating the scope of the heart failure epidemic. Jama 2003, 289(2):194-202.

21. Pritchett AM, Mahoney DW, Jacobsen SJ, Rodeheffer RJ, Karon BL, Redfield MM: Diastolic dysfunction and left atrial volume: a populationbased study. J Am Coll Cardiol 2005, 45(1):87-92.

22. Barnes ME, Miyasaka Y, Seward JB, Gersh BJ, Rosales AG, Bailey KR, Petty GW, Wiebers DO, Tsang TS: Left atrial volume in the prediction of first ischemic stroke in an elderly cohort without atrial fibrillation. Mayo Clin Proc 2004, 79(8):1008-14.

23. Tsang TS, Abhayaratna WP, Barnes ME, Miyasaka Y, Gersh BJ, Bailey KR, Cha SS, Seward JB: Prediction of cardiovascular outcomes with left atrial size: is volume superior to area or diameter? J Am Coll Cardiol 2006, 47(5):1018-23.

24. Tsang TS, Barnes ME, Bailey KR, Leibson CL, Montgomery SC, Takemoto Y, Diamond PM, Marra MA, Gersh BJ, Wiebers DO, Petty GW, Seward JB: Left atrial volume: important risk marker of incident atrial fibrillation in 1655 older men and women. Mayo Clin Proc 2001, 76(5):467-75.

25. Tsang TS, Gersh BJ, Appleton CP, Tajik AJ, Barnes ME, Bailey KR, Oh JK, Leibson C, Montgomery SC, Seward JB: Left ventricular diastolic dysfunction as a predictor of the first diagnosed nonvalvular atrial fibrillation in 840 elderly men and women. J Am Coll Cardiol 2002, 40(9):1636-44.

26. Vaziri SM, Larson MG, Benjamin EJ, Levy D: Echocardiographic predictors of nonrheumatic atrial fibrillation. The Framingham Heart Study. Circulation 1994, 89(2):724-30

27. Tsang TS, Barnes ME, Abhayaratna WP, Cha SS, Gersh BJ, Langins AP Green TD, Bailey KR, Miyasaka Y, Seward JB: Effects of quinapril on left atrial structural remodeling and arterial stiffness. Am J Cardiol 2006, 97(6):916-20.

28. Psaty BM, Manolio TA, Kuller LH, Kronmal RA, Cushman M, Fried LP, White R, Furberg CD, Rautaharju PM: Incidence of and risk factors for atrial fibrillation in older adults. Circulation 1997, 96(7):2455-61.

29. Benjamin EJ, D'Agostino RB, Belanger AJ, Wolf PA, Levy D: Left atrial size and the risk of stroke and death. The Framingham Heart Study. Circulation 1995, 92(4):835-41.

30. Takemoto Y, Barnes ME, Seward JB, Lester SJ, Appleton CA, Gersh BJ, Bailey KR, Tsang TS: Usefulness of left atrial volume in predicting first congestive heart failure in patients $>$ or $=65$ years of age with wellpreserved left ventricular systolic function. Am J Cardiol 2005, 96(6):832-6.

31. Gottdiener JS, Kitzman DW, Aurigemma GP, Arnold AM, Manolio TA: Left atrial volume, geometry, and function in systolic and diastolic heart failure of persons $>$ or $=65$ years of age (the cardiovascular health study). Am J Cardiol 2006, 97(1):83-9.

32. Rossi A, Cicoira M, Zanolla L, Sandrini R, Golia G, Zardini P, EnriquezSarano M: Determinants and prognostic value of left atrial volume in patients with dilated cardiomyopathy. J Am Coll Cardiol 2002, 40(8):1425.

33. Moller JE, Hillis GS, Oh JK, Seward JB, Reeder GS, Wright RS, Park SW, Bailey KR, Pellikka PA: Left atrial volume: a powerful predictor of survival after acute myocardial infarction. Circulation 2003, 107(17):2207-12.

34. Beinart R, Boyko V, Schwammenthal E, Kuperstein R, Sagie A, Hod H, Matetzky S, Behar S, Eldar M, Feinberg MS: Long-term prognostic 
significance of left atrial volume in acute myocardial infarction. J Am Coll Cardiol 2004, 44(2):327-34.

35. Giannuzzi P, Temporelli PL, Bosimini E, Silva P, Imparato A, Corra U, Galli M, Giordano A: Independent and incremental prognostic value of Dopplerderived mitral deceleration time of early filling in both symptomatic and asymptomatic patients with left ventricular dysfunction. J Am Coll Cardiol 1996, 28(2):383-90.

36. Irzmanski R, Banach M, Piechota M, Kowalski J, Barylski M, Cierniewski C, Pawlicki L: Atrial and brain natriuretic peptide and endothelin-1 concentration in patients with idiopathic arterial hypertension: the dependence on the selected morphological parameters. Clin Exp Hypertens 2007, 29(3):149-64.

doi:10.1186/1532-429X-13-42

Cite this article as: Kaminski et al: Strong cardiovascular prognostic implication of quantitative left atrial contractile function assessed by cardiac magnetic resonance imaging in patients with chronic

hypertension. Journal of Cardiovascular Magnetic Resonance 2011 13:42.

\section{Submit your next manuscript to BioMed Central} and take full advantage of:

- Convenient online submission

- Thorough peer review

- No space constraints or color figure charges

- Immediate publication on acceptance

- Inclusion in PubMed, CAS, Scopus and Google Scholar

- Research which is freely available for redistribution

Submit your manuscript at www.biomedcentral.com/submit 